\title{
The neutrophil protein S100A12 is associated with a comprehensive ultrasonographic synovitis score in a longitudinal study of patients with rheumatoid arthritis treated with adalimumab
}

\author{
Hilde Haugedal Nordal 1,3*, Johan G Brun²,3, Anne-Kristine Halse ${ }^{2,3}$, Roland Jonsson ${ }^{1,3}$, Magne K Fagerhol ${ }^{4}$
} and Hilde Berner Hammer ${ }^{5}$

\begin{abstract}
Background: The calcium-binding protein S100A12 correlates with measures of disease activity in patients with rheumatoid arthritis (RA). The protein reflects neutrophil activation and the present objective was to explore in a pilot study the associations between S100A12 and other inflammatory markers, clinical assessments as well as degree of synovitis detected by a comprehensive ultrasonography (US) examination in RA patients during biologic treatment.

Methods: Twenty patients with RA were examined clinically and by use of US as well as laboratory markers S100A12, calprotectin, C-reactive protein (CRP) and erythrocyte sedimentation rate (ESR) before starting adalimumab, with follow-up after 1, 3, 6 and 12 months. Ultrasonographic B-mode (BM) and power Doppler (PD) assessments of 78 joints, 36 tendons/tendon groups and 2 bursas were performed, and sum US scores calculated. Wilcoxon signed rank test assessed treatment response and Spearman rank correlation test was used to calculate correlations.

Results: The concentrations of S100A12 decreased after 3 months $(p<0.01)$ and significant correlations were found between S100A 12 and the other laboratory markers during follow-up $(0.50-0.62, p<0.05)$. Of the clinical assessments, S100A12 had highest correlations with the assessor's global VAS $(0.46-0.85, \mathrm{p}<0.05)$. Compared with CRP and ESR, S100A12 showed higher correlations with the sum US scores (both BM and PD), with median (range) correlation coefficients of 0.55 (0.35-0.78 (NS-p < 0.001)) for sum BM scores and 0.45 (0.27-0.75 (NS-p< 0.001)) for sum PD scores.
\end{abstract}

Conclusions: The S100A12 protein was significantly associated with other inflammatory markers, clinical assessments as well as sum US scores, indicating that S100A12 is a potential marker of inflammation in RA patients.

Keywords: S100 proteins, S100A12, Ultrasonography, Rheumatoid arthritis, Inflammation, Biologic therapy

\section{Background}

Rheumatoid arthritis (RA) is characterized by synovitis that untreated may lead to joint damage and functional disability. Even in clinical remission, a majority of patients with RA had ongoing synovial inflammation as detected by magnetic resonance imaging (MRI) or ultrasonography (US) [1]. Use of these sensitive imaging modalities is expensive or time consuming, thus sensitive biomarkers

\footnotetext{
* Correspondence: Hilde.Nordal@k2.uib.no

'Broegelmann Research Laboratory, Department of Clinical Science, University of Bergen, Postboks 7804, N-5020 Bergen, Norway

${ }^{3}$ Department of Rheumatology, Haukeland University Hospital, Bergen, Norway Full list of author information is available at the end of the article
}

reflecting the ongoing joint inflammation may be of considerable value for clinical decisions.

S100A12 is a calcium-binding protein, expressed predominantly in neutrophils, but also in monocytes $[2,3]$. It is released from activated neutrophils [4] and has proinflammatory effects on endothelium and immune cells that have been summarized in a review article [5].The protein was up-regulated in synovial fluid in RA patients compared with patients with osteoarthritis [6], and it differentiated RA from other forms of inflammatory arthritis when exploring synovial fluid [7]. S100A12 was increased in serum of RA patients compared with healthy controls, and was associated with the presence of rheumatoid factor 
(RF) and anti-citrullinated peptide antibodies (ACPA) [8]. Both in synovial fluid and in serum elevated levels of S100A12 were found in erosive forms of RA compared with non-erosive forms [9]. S100A12 was found in inflamed synovia of RA patients, but it was not expressed in synovial tissue without inflammation [10]. Serum concentrations of S100A12 correlated with synovial fluid levels and with measures of disease activity as erythrocyte sedimentation rate (ESR) and Ritchie articular index [10]. S100A12 serum concentrations were also elevated in RA patients before treatment with intra-articular corticosteroids or anti-tumour necrosis factor (anti-TNF), and decreased significantly in patients who responded to these treatments [11]. S100A12 expression in synovia was also reduced in these two groups after treatment [11]. Although serum levels of S100A12 are increased in RA patients compared with controls $[8,10]$, the upper normal level in healthy persons has yet to be defined.

US is a sensitive imaging modality for detecting synovitis in RA patients. The amount of synovitis is detected by $\mathrm{B}$-mode (BM). The degree of vascularization, reflecting the inflammatory activity, is evaluated by use of power Doppler (PD). A comprehensive US examination of joints and tendons of RA patients may thus be able to reflect the total amount of ongoing inflammation in these structures.

The major leukocyte S100 protein, calprotectin (heterocomplex of S100A8/A9), was investigated earlier in plasma samples of this cohort and correlated with the US sum scores [12]. The present pilot study explores the associations between serum levels of S100A12 and laboratory and clinical assessments of disease activity, as well as US sum scores from a comprehensive examination of joints and tendons in a longitudinal follow-up of RA patients starting biologic treatment.

\section{Methods \\ Patients}

Twenty patients (15 women and 5 men) with RA according to the American Rheumatism Association 1987 revised criteria [13] with median (range) age 53 (21 to 78) years and disease duration 7.5 (1 to 26$)$ years were included as described elsewhere [14]. A total of 70\% were IgM RF positive and 80\% ACPA positive. At inclusion all patients used methotrexate as disease-modifying anti-rheumatic drug (DMARD) (median (range) dose 17.5 (7.5-25) mg per week), 70\% used prednisolone (median (range) dose 7.5 (3.75-15) mg per day) and two patients were on daily non-steroidal anti-inflammatory drugs (NSAIDs). Median (range) DAS28 was 5.3 (3.47.7) at baseline. The patients were included the day they started treatment with adalimumab $40 \mathrm{mg}$ every second week as their first biologic agent. The study was approved by the Regional Committee for Medical and
Health Research Ethics, South-East (REK), and the patients gave written consent according to the Declaration of Helsinki.

\section{Laboratory analyses}

Blood samples were drawn at inclusion and after 1, 3, 6 and 12 months of treatment with adalimumab. Conventional inflammatory markers included ESR and CRP, analysed by use of in-house methodology, with upper normal levels of $20 \mathrm{~mm} / \mathrm{h}$ for ESR and $4 \mathrm{mg} / \mathrm{L}$ for CRP. Calprotectin in plasma was examined by use of enzyme-linked immunosorbent assay (ELISA) kits from CALPRO AS, Norway. Sera from all five examinations were frozen at $-70^{\circ} \mathrm{C}$. S100A12 concentrations in serum were determined by ELISA as follows: 96-well high binding Costar $3590 \mathrm{mi}-$ cro plates from Costar Corporation, USA, were coated with mouse monoclonal anti-S100A12 $5 \mu \mathrm{g} / \mathrm{mL}$ in $0.1 \mathrm{M}$ sodium citrate $\mathrm{pH} 6,150 \mu \mathrm{L}$ per well, for $18 \mathrm{~h}$ to 4 weeks at $+5^{\circ} \mathrm{C}$. Samples were diluted 1:5 using the sample dilution buffer. After washing the wells three times, $50 \mu \mathrm{L}$ of calibrators or samples were added in triplicate wells and incubated at room temperature with shaking (500 rpm) for $40 \mathrm{~min}$. A second monoclonal was conjugated with alkaline phosphatase. After washing again, all wells were added $50 \mu \mathrm{L}$ of the ALP conjugate diluted 1:1000 and incubated again for $40 \mathrm{~min}$. After a final wash, all wells were given $100 \mu \mathrm{L}$ ALP substrate. Reading at $405 \mathrm{~nm}$ was performed when the optical density (OD) of the higher standard $(512 \mathrm{ng} / \mathrm{mL})$ was between 2.0 and 3.0. For samples with concentrations of 512 and $16 \mathrm{ng} / \mathrm{mL}$ the intra-assay coefficients of variation were $6.5 \%$ and $10.6 \%$ and interassay coefficients of variation were $2.3 \%$ and $10.9 \%$ respectively. The lower limit of quantification of the ELISA was $4 \mathrm{ng} / \mathrm{mL}$. The reactivity and specificity of the antibodies were previously tested by ELISA using micro wells coated with S100A12 or calprotectin, which has considerable amino acid sequence homology to S100A12 [15].

\section{Clinical assessments}

Forty joints (proximal inter-phalangeals 1-5, metacarpophalangeals $1-5$, wrists, elbows, shoulders, knees, ankles and metatarso-phalangeals 1-5) were assessed by one of two experienced nurses for tenderness and swelling, blinded for the results of the US examination. They also scored the global disease activity on a visual analogue scale (assessor's global VAS). The disease activity score DAS28 [16] was calculated.

\section{Ultrasonography}

US examinations were performed by one experienced sonographer $(\mathrm{HBH}$ using a Siemens Antares Sonoline machine; Siemens Medical Solutions, California, USA) at the day of inclusion and after 1, 3, 6 and 12 months as previously described [12,14]. A total of 78 joints and 36 


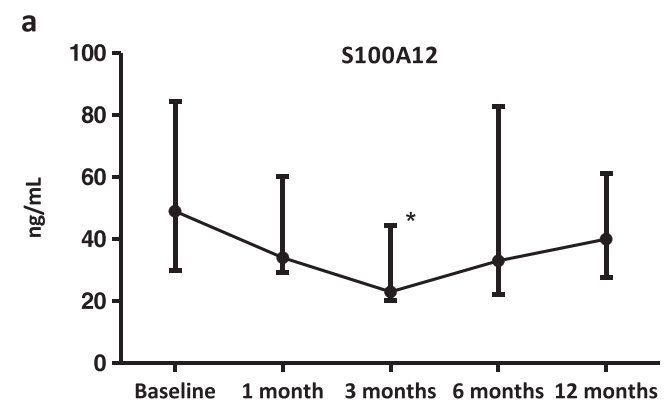

b

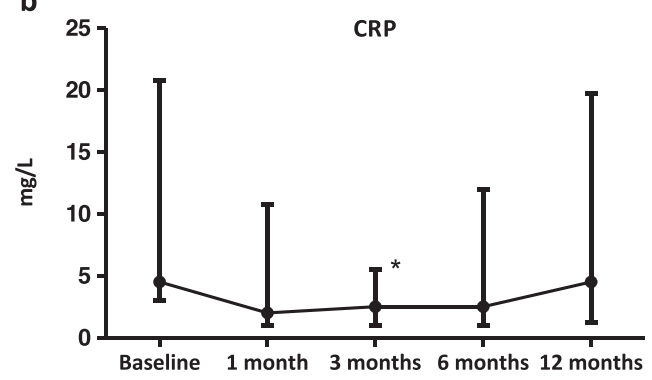

C

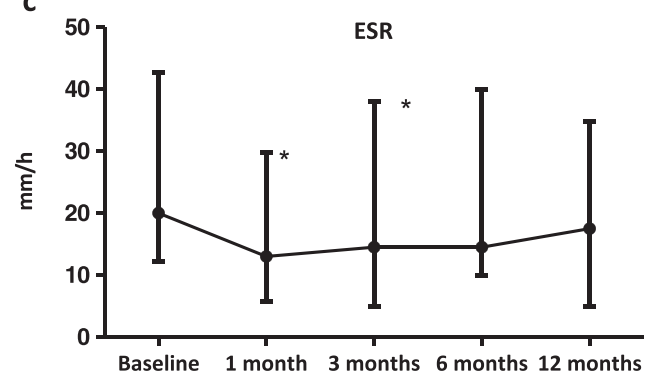

d
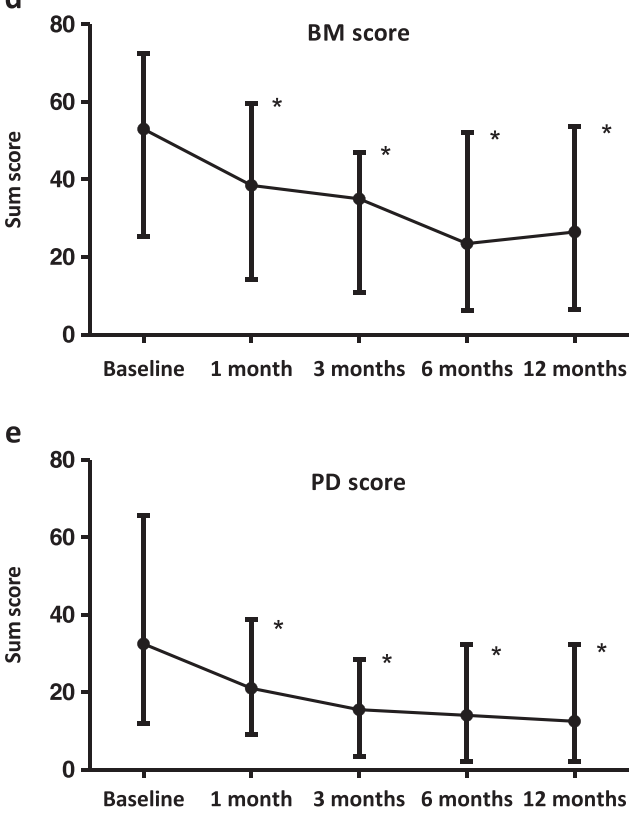

Figure 1 Median levels (interquartile range) of the inflammatory markers and ultrasonographic scores during 12 months follow-up. a) Concentrations of S100A12. b) Concentrations of C-reactive protein (CRP). c) Erythrocyte sedimentation rates (ESRs). d) B-mode (BM) sum scores. e) Power Doppler (PD) sum scores. *Significantly decreased levels from baseline, $\mathrm{p}<0.01$.

tendons or tendon groups as well as the bilateral subdeltoid bursae were assessed for arthritis, tenosynovitis and bursitis by grey scale or B-mode (BM) presence of synovial hypertrophy and fluid (scored together) and presence of power Doppler (PD) vascularization, both scored on a 4 -point scale $(0=$ no, $1=$ minor, $2=$ moderate, $3=$ major presence). The US examiner had no access to US results from the previous examinations and was blinded for the results from the clinical joint assessments and laboratory tests completed the same day.

\section{Statistics}

Wilcoxon signed rank test was used to analyse the differences between the levels of S100A12, ESR, CRP and US scores before starting adalimumab treatment and during follow-up. Associations between laboratory markers, clinical evaluations and US scores were analysed by use of Spearman rank order correlations. IBM SPSS Statistics version 20 was used for the statistical analyses and $\mathrm{p}<$ 0.05 was considered statistically significant. All tests for significance were two-sided.

\section{Results}

The patients had median (range) S100A12 levels of 49 (18 to 475$) \mathrm{ng} / \mathrm{mL}$ at baseline, 34 (18 to 687$) \mathrm{ng} / \mathrm{mL}$ after 1 month, 23 (16 to 173$) \mathrm{ng} / \mathrm{mL}$ after 3 months, 33 (19 to 394) $\mathrm{ng} / \mathrm{mL}$ after 6 months and 40 (12 to 620$) \mathrm{ng} / \mathrm{mL}$ after 12 months. The level of S100A12, ESR and CRP changed significantly from baseline to 3 months follow-up and ESR already after 1 month, while the sum US scores decreased significantly during follow-up (Figure 1).

There were significant correlations between S100A12 levels and sum US scores of all joints, tendons and bursae for BM and PD at baseline, after one month and 6 months. During the study, S100A12 showed higher correlation coefficients with the US scores than the conventional inflammatory parameters CRP and ESR (Table 1). The correlations between S100A12 and the inflammatory markers, as well as clinical assessments, are shown in Table 2. Closest associations were found between S100A12 and CRP/assessor's global VAS. During followup, different degree of correlation was found between the leukocyte proteins S100A12 and calprotectin, from not significant to moderately significant correlations. 
Table 1 Spearman rank correlation coefficients between inflammatory markers and sum BM/PD scores

\begin{tabular}{lccccc}
\hline & Baseline & 1 month & 3 months & 6 months & 12 months \\
\hline S100A12 & $0.78^{* * *} / 0.75^{* * *}$ & $0.68^{* * *} / 0.65^{* * *}$ & $0.35 / 0.27$ & $0.55^{*} / 0.45^{*}$ & $0.43 / 0.43$ \\
ESR & $0.37 / 0.48^{*}$ & $0.36 / 0.35$ & $0.45^{*} / 0.42$ & $0.32 / 0.33$ & $0.35 / 0.40$ \\
CRP & $0.33 / 0.34$ & $0.04 / 0.11$ & $0.49^{*} / 0.35$ & $0.59^{* *} / 0.43$ & $0.57^{* *} / 0.55^{*}$ \\
\hline
\end{tabular}

$\mathrm{CRP}=\mathrm{C}$-reactive protein, $\mathrm{ESR}=$ erythrocyte sedimentation rate, $\mathrm{BM}=\mathrm{B}$-mode, $\mathrm{PD}=$ power Doppler, ${ }^{*} \mathrm{p}<0.05,{ }^{* *} \mathrm{p}<0.01,{ }^{* * *} \mathrm{p}<0.001$.

\section{Discussion}

The present one-year follow-up study of RA patients starting treatment with adalimumab is the first to analyse the associations between serum levels of S100A12 and a comprehensive US joint examination, and significant correlations were currently found.

All the inflammatory laboratory markers decreased during the study. However, they tended to increase at 6 months onward (Figure 1), which could indicate reduced efficacy of the medication. It could be speculated that it may be caused by development of antibodies against adalimumab in some of the patients [17]. On the other hand, this tendency was not seen for the US sum scores [14]. A possible explanation could be that rise in laboratory markers may be seen before changes in US scores. It could also be speculated that, in parallel with findings which suggest a possible dissociation between disease activity and radiographic damage [18], there could be a dissociation between the disease activity as measured by inflammatory markers and US findings in some patients treated with a biologic agent.

The S100A12 serum levels were lower in this study than those reported for RA patients with active arthritis $[10,11]$. The decrease following successful therapy was also less pronounced than what has been reported previously [11]. This might be explained by the use of a different ELISA-test and thus the levels cannot be directly compared. In addition, the levels of the other inflammatory markers were rather low, even at baseline.

S100A12 correlated better and more consistently with the comprehensive US sum BM and PD scores than the conventional inflammatory parameters (Table 1). However, this might be partly explained by the low levels of the conventional parameters, at least for CRP having a median level of $4.5 \mathrm{mg} / \mathrm{L}$ at baseline. US is a sensitive modality for detection of synovitis, and thus the present findings indicate that the neutrophil protein S100A12 is a biomarker of synovial inflammation in RA. Presently small and large joints were equally weighted in the US scores, and thus not exactly reflecting the total amount of synovitis. A larger study should include a weighting of joints taking into account the size of the inflamed synovium. This might be a more accurate way of assessing the associations between US and the leukocyte proteins calprotectin and S100A12.

Although both S100A12 and calprotectin are released by neutrophil activation, their correlations during followup were presently found to be variable. S100A12 was not superior to calprotectin as a biomarker for RA in this cohort, but the associations between these two S100 proteins and clinical assessments and US scores should be explored in further studies. Also S100A12's ability to predict destruction should be assessed in larger cohorts of RA patients.

In a large cohort of patients with juvenile idiopathic arthritis it was found that increased levels of S100A12 and calprotectin, in addition to high-sensitivity C-reactive protein, indicated subclinical inflammation and thereby could identify patients with increased risk of relapse when in clinical remission [19]. Whether this is paralleled in RA, remains to be shown. Many RA patients in clinical remission have remaining inflammatory activity of their joints detected by US [1]. In our cohort a significantly higher number of inflamed joints were found by US than by clinical assessments at all the examinations [14]. Serum levels of S100A12 may be of help to identify RA patients with remaining inflammatory activity of joints.

The present study has several strengths. Primarily, this is the first study exploring the associations between S100A12 serum levels and a comprehensive US examination. In

Table 2 Spearman rank correlation coefficients between S100A12 and inflammatory markers/clinical evaluations of disease activity

\begin{tabular}{lccccc}
\hline & Baseline & 1 month & 3 months & 6 months & 12 months \\
\hline ESR & $0.50^{*}$ & 0.29 & 0.25 & 0.38 & $0.62^{* *}$ \\
CRP & $0.61^{* *}$ & 0.40 & $0.50^{*}$ & $0.66^{* *}$ & $0.59^{* *}$ \\
Calprotectin & $0.56^{*}$ & 0.34 & 0.12 & $0.52^{*}$ & $0.57^{* *}$ \\
Assessor's global VAS & $0.85^{* * *}$ & $0.58^{* *}$ & 0.12 & $0.52^{*}$ & $0.46^{*}$ \\
Swollen joints (of 40) & 0.41 & $0.45^{*}$ & 0.33 & 0.31 & $0.48^{*}$ \\
DAS28 & 0.39 & 0.21 & 0.23 & 0.34 & $0.51^{*}$ \\
\hline
\end{tabular}

$\mathrm{ESR}=$ erythrocyte sedimentation rate, $\mathrm{CRP}=\mathrm{C}$-reactive protein, VAS $=$ visual analogue scale, DAS28 $=$ disease activity score of 28 joints, ${ }^{*} \mathrm{p}<0.05,{ }^{* *} \mathrm{p}<0.01,{ }^{* * *} \mathrm{p}<0.001$. 
addition, it is a longitudinal 12 months follow-up study, giving the possibility to study changes in S100A12 during treatment with biologic medication. The weakness of this pilot study is the limited number of patients included, which reduces the strength of the present findings.

\section{Conclusions}

In this pilot study of $20 \mathrm{RA}$ patients followed for 12 months after initiation of adalimumab treatment, serum levels of S100A12 were associated with clinical and laboratory assessments as well as with a comprehensive ultrasonographic examination. This indicates that S100A12 could be a biomarker of synovitis in RA, and these promising findings should be explored further in larger cohorts of RA patients to evaluate S100A12 as a marker of inflammation.

\begin{abstract}
Abbreviations
ACPA: Anti-citrullinated antibodies; BM: B-mode or grey scale; CRP: C-reactive protein; DAS28: Disease activity score of 28 joints; ELISA: Enzyme-linked immunosorbent assay; ESR: Erythrocyte sedimentation rate; MRI: Magnetic resonance imaging; PD: Power Doppler; RA: Rheumatoid arthritis;

TNF: Tumour necrosis factor; US: Ultrasonography; VAS: Visual analogue scale.
\end{abstract}

\section{Competing interests}

The authors declare that they have no competing interests.

\section{Authors' contributions}

$\mathrm{HHN}$ took part in the study concept and design, was responsible for the S100A12 analyses, the analysis and interpretation of data and drafting of the manuscript. JGB, AKH and RJ took part in the study concept and design, interpretation of data and drafting of the manuscript. MKF took part in the study concept and design, was responsible for the development of the S100A12 ELISA and read the manuscript critically. $\mathrm{HBH}$ took part in the study concept and design, performed the ultrasonographic examinations and took part in drafting of the manuscript. All authors read and approved the final manuscript.

\section{Acknowledgements}

We thank Marianne Eidsheim for excellent technical assistance with the S100A12 ELISA and Anne Katrine Kongtorp and Margareth Sveinsson for the clinical assessments. HBH was supported by an unrestricted grant from Abbott Norway. The study was supported by Broegelmann Foundation.

\section{Author details}

${ }^{1}$ Broegelmann Research Laboratory, Department of Clinical Science, University of Bergen, Postboks 7804, N-5020 Bergen, Norway. ${ }^{2}$ Section for Rheumatology, Department of Clinical Science, University of Bergen, Bergen, Norway. ${ }^{3}$ Department of Rheumatology, Haukeland University Hospital, Bergen, Norway. ${ }^{4}$ Prof. Fagerhol's Research Laboratory, Oslo, Norway. ${ }^{5}$ Department of Rheumatology, Diakonhjemmet Hospital, Oslo, Norway.

Received: 8 July 2014 Accepted: 26 September 2014 Published: 4 October 2014

\section{References}

1. Brown AK, Quinn MA, Karim Z, Conaghan PG, Peterfy CG, Hensor E, Wakefield RJ, O'Connor PJ, Emery P: Presence of significant synovitis in rheumatoid arthritis patients with disease-modifying antirheumatic drug-induced clinical remission: evidence from an imaging study may explain structural progression. Arthritis Rheum 2006, 54(12):3761-3773.

2. Guignard F, Mauel J, Markert M: Identification and characterization of a novel human neutrophil protein related to the S100 family. Biochem J 1995, 309(Pt 2):395-401.

3. Robinson MJ, Hogg N: A comparison of human S100A12 with MRP-14 (S100A9). Biochem Biophys Res Commun 2000, 275(3):865-870.

4. Boussac M, Garin J: Calcium-dependent secretion in human neutrophils: a proteomic approach. Electrophoresis 2000, 21(3):665-672.
5. Foell $\mathrm{D}$, Roth J: Proinflammatory $\mathrm{S} 100$ proteins in arthritis and autoimmune disease. Arthritis Rheum 2004, 50(12):3762-3771.

6. Rouleau P, Vandal K, Ryckman C, Poubelle PE, Boivin A, Talbot M, Tessier PA: The calcium-binding protein S100A12 induces neutrophil adhesion, migration, and release from bone marrow in mouse at concentrations similar to those found in human inflammatory arthritis. Clin Immuno/ 2003, 107(1):46-54.

7. Baillet A, Trocme C, Berthier S, Arlotto M, Grange L, Chenau J, Quetant S, Seve M, Berger F, Juvin R, Morel F, Gaudin P: Synovial fluid proteomic fingerprint: S100A8, S100A9 and S100A12 proteins discriminate rheumatoid arthritis from other inflammatory joint diseases.

Rheumatology (Oxford) 2010, 49(4):671-682.

8. Chen YS, Yan W, Geczy CL, Brown MA, Thomas R: Serum levels of soluble receptor for advanced glycation end products and of S100 proteins are associated with inflammatory, autoantibody, and classical risk markers of joint and vascular damage in rheumatoid arthritis. Arthritis Res Ther 2009, 11(2):R39.

9. Liao H, Wu J, Kuhn E, Chin W, Chang B, Jones MD, O'Neil S, Clauser KR, Karl J, Hasler F, Roubenoff R, Zolg W, Guild BC: Use of mass spectrometry to identify protein biomarkers of disease severity in the synovial fluid and serum of patients with rheumatoid arthritis. Arthritis Rheum 2004, 50(12):3792-3803.

10. Foell D, Kane D, Bresnihan B, Vogl T, Nacken W, Sorg C, Fitzgerald O, Roth J: Expression of the pro-inflammatory protein S100A12 (EN-RAGE) in rheumatoid and psoriatic arthritis. Rheumatology (Oxford) 2003, 42(11):1383-1389.

11. Wittkowski H, Foell D, af Klint E, De Rycke L, De Keyser F, Frosch M, Ulfgren AK, Roth J: Effects of intra-articular corticosteroids and anti-TNF therapy on neutrophil activation in rheumatoid arthritis. Ann Rheum Dis 2007, 66(8):1020-1025.

12. Hammer HB, Fagerhol MK, Wien TN, Kvien TK: The soluble biomarker calprotectin (a S100 protein) is associated to ultrasonographic synovitis scores and is sensitive to change in patients with rheumatoid arthritis treated with adalimumab. Arthritis Res Ther 2011, 13(5):R178.

13. Arnett FC, Edworthy SM, Bloch DA, McShane DJ, Fries JF, Cooper NS, Healey LA, Kaplan SR, Liang MH, Luthra HS, Medsger TA Jr, Mitchell DM, Neustadt DH, Pinals RS, Schaller JG, Sharp JT, Wilder RL, Hunder GG: The American Rheumatism Association 1987 revised criteria for the classification of rheumatoid arthritis. Arthritis Rheum 1988, 31(3):315-324.

14. Hammer HB, Sveinsson M, Kongtorp AK, Kvien TK: A 78-joints ultrasonographic assessment is associated with clinical assessments and is highly responsive to improvement in a longitudinal study of patients with rheumatoid arthritis starting adalimumab treatment. Ann Rheum Dis 2010, 69(7):1349-1351.

15. Fagerhol MK, Larsen A, Brun JG, Hammer HB, Angel K, Kvien TK, Kinne I, Madland TM: Large molecular size EDTA-resistant complexes containing S100A12, ERAC, in serum during inflammatory conditions. Scand J Clin Lab Invest 2012, 72(2):129-136.

16. Prevoo ML, Hof MA V't, Kuper $H H$, van Leeuwen MA, van de Putte LB, van Riel PL: Modified disease activity scores that include twenty-eight-joint counts. Development and validation in a prospective longitudinal study of patients with rheumatoid arthritis. Arthritis Rheum 1995, 38(1):44-48.

17. Bartelds GM, Wijbrandts CA, Nurmohamed MT, Stapel S, Lems WF, Aarden L, Dijkmans BA, Tak PP, Wolbink GJ: Clinical response to adalimumab: relationship to anti-adalimumab antibodies and serum adalimumab concentrations in rheumatoid arthritis. Ann Rheum Dis 2007, 66(7):921-926

18. Keystone EC: Clinical implications of understanding radiographic findings in relation to clinical outcomes in rheumatoid arthritis. J Rheumatol Suppl 2009, 82:11-16.

19. Gerss J, Roth J, Holzinger D, Ruperto N, Wittkowski H, Frosch M, Wulffraat N, Wedderburn L, Stanevicha V, Mihaylova D, Harjacek M, Len C, Toppino C, Masi M, Minden K, Saurenmann T, Uziel Y, Veseley R, Apaz MT, Kuester R-M, Elorduy MJ, Burgos-Vargas R, loseliani M, Magni-Manzoni S, Unsal E, Anton J, Balogh Z, Hagelberg S, Mazur-Zielinska H, Tauber T, et al: Phagocyte-specific S100 proteins and high-sensitivity $C$ reactive protein as biomarkers for a riskadapted treatment to maintain remission in juvenile idiopathic arthritis: a comparative study. Ann Rheum Dis 2012, 71(12):1991-1997.

doi:10.1186/1471-2474-15-335

Cite this article as: Nordal et al:: The neutrophil protein S100A12 is associated with a comprehensive ultrasonographic synovitis score in a longitudinal study of patients with rheumatoid arthritis treated with adalimumab. BMC Musculoskeletal Disorders 2014 15:335. 